\title{
Quality Control Strategy for Automated CBC: A Laboratory Point of View Deducted from an Internal Study Organised in an Emergency Laboratory
}

\author{
Oana Roxana Oprea ${ }^{1,2}$, Adina Hutanu ${ }^{1,3^{*}}$, Oana Pavelea ${ }^{2}$, David Robert Kodori ${ }^{2}$, \\ Minodora Dobreanu ${ }^{1,3}$ \\ 1. Department of Laboratory Medicine, George Emil Palade University of Medicine, Pharmacy, \\ Science, and Technology of Targu Mures, Romania \\ 2. Emergency Clinical County Hospital Targu Mures, Romania \\ 3. Center for Advanced Medical and Pharmaceutical Research, George Emil Palade University of \\ Medicine, Pharmacy, Science, and Technology of Targu Mures, Romania
}

\begin{abstract}
Introduction: The aim of this study was to determine the performance of the total testing process of complete blood count (CBC) on two different instruments in an emergency setting of a county hospital, and to design an appropriate internal quality control plan. Materials and method: Two models of Statistical Quality Control (SQC) were evaluated on Sysmex XT-1800i and Cell-Dyne Ruby: 3 levels of commercial blood every 8 hours (N=9) and an alternative model using 3 levels every 12 hours $(N=6)$ as shift changes. Total Error (TE) was calculated using the formula: $T E=B i a s \%+1.65 x C V \%$; Sigma score was calculated using the formula: Sigma=[(TEa\%-Bias\%]/ $C V \%$. Values for coefficient of variation (CV\%) and standard deviation (SD) were obtained from laboratory data and Bias\% from proficiency testing. For the pre-analytical phase Sigma score was calculated, while for post-analytical phase the turnaround time (TAT) was assessed. Results: TE for all directly measured parameters, for both instruments, had lower values than Total Error allowable (TEa). CV\% for almost all parameters had lower values than CV\% derived from biological variation except for platelets (PLT) at low level on Sysmex XT-1800i and red blood cells (RBC) on Cell-Dyne Ruby. Sigma score ranged from as low as 2 to 10. Sigma score for pre-analytical phase was 4.2 and turnaround time was 36 minutes on average. Conclusions: Given the performances of the total testing process implemented for $C B C$ in our laboratory, performing the internal control after every 50 samples/ batch seems to fulfill both the Health Ministry Order (HMO) 1301/2007 and International Organization for Standardization ISO 15189:2013 recommendation. All quality instruments must work together to assure better patient results and every laboratory should design its own control plan that is appropriate for better quality achievement.
\end{abstract} Keywords: quality control, Six-Sigma, moving average, Total Error, Bull's algorithm Received: $5^{\text {th }}$ September 2019; Accepted: 22 ${ }^{\text {nd }}$ January 2020; Published: $23^{\text {th }}$ January 2020

*Corresponding author: Adina Hutanu, George Emil Palade University of Medicine, Pharmacy, Science, and Technology of Targu Mures, Romania. E-mail: adina.hutanu@umfst.ro 


\section{Introduction}

Although Statistical Quality Control (SQC) design has been used for decades as quality control (QC) tool, some questions remain unanswered. The number of levels of control that must be used in a laboratory for automated complete blood count (CBC) hematology testing is traditionally set to three with low, normal, and high levels, and for most of the parameters this satisfies the requirements of ISO 15189:2013 Standard (1). Although using three levels of control complies with the requirements, these may not be sufficient. According to the published decision limits for some parameters in hematology, there are 4 decision limits (2) that cannot be covered by the three levels of traditionally used controls. Concerning the frequency of these controls, there are no regulations that can be widely applied. According to Clinical Laboratory Improvement Amendments (CLIA) recommendations, a minimum requirement for the frequency of controls is 24 hours without reference to the case of laboratories that run 24 hours shifts, except for manual methods (3). According to the Romanian Health Ministry Order (HMO) number 1301/2007, laboratories that work 24-hour shifts must run a control every 8 hours, but without any reference regarding the level of these controls. According to ISO 15 189:2013, SQC practices must be designed in a manner that allow error detection in the same run in which that error appears (1). The aim of this study was to determine the performance of the total testing process of CBC using two different models in an emergency setting of a county hospital and to design an appropriate internal QC plan.

\section{Materials and methods}

Statistical quality control (SQC) design implemented in our laboratory follows recommendations of HMO 1301/2007 concerning the frequency of quality control (every 8 hours) and recommendations of Standard ISO 15189:2013, in terms of three levels control. In order to comply with both regulations, every 24 hours, 9 control points are determined (3x3 model, 3 times/day X 3 control levels $\mathrm{N}=9$ ) using stabilized commercially control blood. Commercial stabilized blood is produced for each of the two instruments, Sysmex XT 1800i (Sysmex Corporation, Japan) and Cell-Dyne Ruby (Abbott, Illinois, USA) .

To establish the performance of used methods, total error of the method (TE) and Sigma value for directly measured parameters were assessed. For estimation of TE value, the formula based on Bias and coefficient of variation $(\mathrm{CV} \%)$ was used: TE $=$ Bias $\%+1.65$ x CV\% (4). For an accurate estimation of Bias, data from proficiency testing programs were used (5). For each instrument, three samples representing survey materials (with normal, low, and high values) were run 13 times and the average of these values (for each level) was used as laboratory reported value to proficiency testing provider. Percentage Bias \% was calculated using laboratory reported values and mean value for peer group of the instrument provided from the PT (proficiency testing) scheme. Additionally, for Cell-Dyne Ruby, Bias\% was calculated using data from interlaboratory control provided by the manufacturer (through StatsLink platform). The CV\% was calculated from three levels of routine internal QC materials mean values and standard deviation (SD) in laboratory conditions. For Cell-Dyne Ruby instrument values for $\mathrm{CV} \%$ from interlaboratory control reports analyzed by StatsLink were also used.

For comparison purposes, in the same period, a different model of SQC design was proposed: three levels of commercial control blood (different vials than those used in routine conditions) were tested two times a day at 12 hours interval ( $2 x$ model, $N=6$ ) for each shift change by the same operators, as routine controls. 
1. All QC materials were tested in "Open Mode" with proper warming and mixing of the vials, according to the manufacturer's instructions. For these controls, Levey-Jennings charts were created using Excel software. For TE and Bias\% estimations, the same formulas as stated above, in the previous model of SQC were used, while mean value, $\mathrm{SD}$, and $\mathrm{CV} \%$ were calculated using Excel Software. The Sigma value was estimated using the formula provided by Westgard (4), where the allowable total error $(\mathrm{TEa} \%)$ value was referred from Clinical Laboratory Improvement Amendments (CLIA) regulations:

$$
\text { Sigma }=(\text { TEa } \%-\text { Bias\% }) / C V \%
$$

2. To supplement data from SQC design using commercial blood and to have a better estimation of the instrument performance, the Moving Average (MoA) function was also used. Cell-Dyne Ruby instrument software offers the possibility to assess the Moving Average function, while for Sysmex XT-1800i analyzer, mean values for red blood cells indices as mean corpuscular volume (MCV), mean corpuscular hemoglobin ( $\mathrm{MCH})$, and mean corpuscular hemoglobin concentration (MCHC) were calculated using Bull's algorithm (6). In order to verify mean values and tolerance limits in the software of Cell-Dyne Ruby instrument for the population addressing our laboratory, 9,000 patient values, received from July to September 2018, were evaluated. Results for red cell indices from these patients were organized in batches of 20 samples to establish the mean values and tolerance limits for the population adressing our laboratory.

3. For assessment of the total testing process, both pre-analytical and post-analytical phases were tracked. Continuous evaluation of sample quality was undertaken, all samples were checked for proper identity (ID), type of tube, a correct proportion between blood and eth- ylene-diamine-tetraacetic acid (EDTA), insufficient sample volume for aspiration, or the presence of clots. Samples with defects were recorded in the Laboratory Informational System (LIS) and Excel workbook. Using the formula for defects per million of opportunities (DPMO), available online as a free calculator on www. westgard.com, the Sigma score was calculated. In 2018, a number of 82,788 tubes for complete blood count were assessed, of which 325 had one or more of the 5 predefined defects. To evaluate proper detection of such samples by the operators, samples with known defects were sent to the laboratory in terms of normal working conditions. These samples (2/day for five working days) were known only by the investigators of this study. Also, the time from which request was created in LIS to the moment the sample was received in the laboratory was evaluated. The turn around time (TAT) was assessed additionally, as a quality indicator. The start of the process was set at the time the CBC tube was received in the laboratory, while the end time was set at the time the results were released in LIS. Tolerance interval was defined as 1 hour.

\section{Statistical analysis}

All calculations and formulas used were executed on Excel spreadsheets, the software of the instrument, and with free online calculators available at www.westgard.com. For CV\% and Bias\% calculations formulas stated in CLSI EP$15 \mathrm{~A} 3$ were used.

\section{Results}

For routine SQC design ( $3 \times 3$ model), the TE\% calculated had smaller values than values of $\mathrm{TEa} \%$ for all parameters analyzed for both instruments. When using CV\% from internal quality control and Bias\% from interlaboratory control data report, TE\% values for Cell-Dyne 
Ruby analyzer were also lower than values accepted for $\mathrm{TEa} \%$. When comparing $\mathrm{CV} \%$ values for each directly measured parameter with $\mathrm{CV} \%$ values from Ricos database for Biological Variation, almost all parameters had lower CV\% values than values stated for Optimal Biological Variation. Parameters with a higher value than accepted for $\mathrm{CV} \%$ values were platelet (PLT) for Sysmex XT-1800i instrument, with CV of $10.19 \%$ (desirable value $9.1 \%$ ) when using $3 \times 3$ SQC design, and $10.59 \%$ when using $2 \times 3$ SQC design (for Low-Level QC).

For the Cell-Dyne Ruby instrument, red blood cells (RBC) CV\% values for Low Level QC were higher than the desirable value $(1.6 \%)$, irrespective of the SQC design used (Table 2c). Additionally, RBC CV\% values were higher than the accepted value for High-Level control material when using 2x3 SQC design.

Sigma values for Sysmex XT-1800i instrument were different for each level or SQC design used: for Normal-Level QC, Sigma values were between 5-8 when using $3 \times 3$ SQC design, and between 5-10 when using 2x3 SQC design. For High-Level QC, Sigma values were between 3-10 when using routine SQC design and between 3-9 when using 2x3 SQC design. For Low-Level QC, Sigma value ranged between 2-7 and 2-10 for routine SQC and 2x3 SQC design, respectively (as shown in figure $1 \mathrm{a}-\mathrm{b}$ ).

For Cell-Dyne Ruby instrument, Sigma values also varied according to the control level as shown in Table 1 (for Sysmex XT-1800i), and Table 2 (for Cell-Dyne Ruby). For Normal-Level control, Sigma values ranged between 4-7 for routine SQC design, between 3-6 for 2x3 SQC design, and between 3-7 when using data from interlaboratory control report. For High-Level QC, Sigma was between 3-6 for routine SQC design, 3-7 for 2x3 SQC design, and 3-8 for interlaboratory control data, while for Low-Level
QC, Sigma values were between 2-6 regardless of the SQC design used.

For the MoA function of the Cell-Dyne Ruby instrument, values obtained for erythrocyte indices were: $\mathrm{MCV}=89.9 \mathrm{fl}, \mathrm{MCH}=29 \mathrm{pg}$, and $\mathrm{MCHC}=32.5 \mathrm{~g} / \mathrm{dl}$, with a tolerance limit of $3 \%$, results that correspond to values implemented by the manufacturer in the instrument software. For Sysmex XT-1800i, values were obtained by implementing Bull's algorithm in Excel Software. Values obtained for erythrocytes indices were: $\mathrm{MCV}=84.4 \mathrm{fl}, \mathrm{MCH}=29.5 \mathrm{pg}, \mathrm{MCHC}=35.0 \mathrm{~g} / \mathrm{dl}$ with a tolerance limit of $3 \%$.

In 2018, the Sigma score obtained for CBC hematology tubes received in our laboratory was 4.2; all samples with known defects being correctly identified by the laboratory personnel. The time from sample request to the time the sample arrived to the laboratory was 36 minutes on average, but in some cases the time was as long as three and a half hours. On average, the TAT from the moment the samples arrived in the laboratory to the time the results were released to the LIS was 13 minutes.

\section{Discussions}

Throughout his mathematical model, Parvin showed that in continuous run mode, bracketed controls increase the error detection, this detection being dependent on the number of controls used and the number of patient samples reported between two control events (7).

In a published Six Sigma estimate on www. westgard.com, precision claims from manufacturers and a Bias assumed as 0 , were used in order to calculate the performance of high volume hematology analyzers. Results obtained in our study revealed that for Low-level QC, both our instruments had poorer performance for PLT and RBC than stated by the manufacturer. Low 

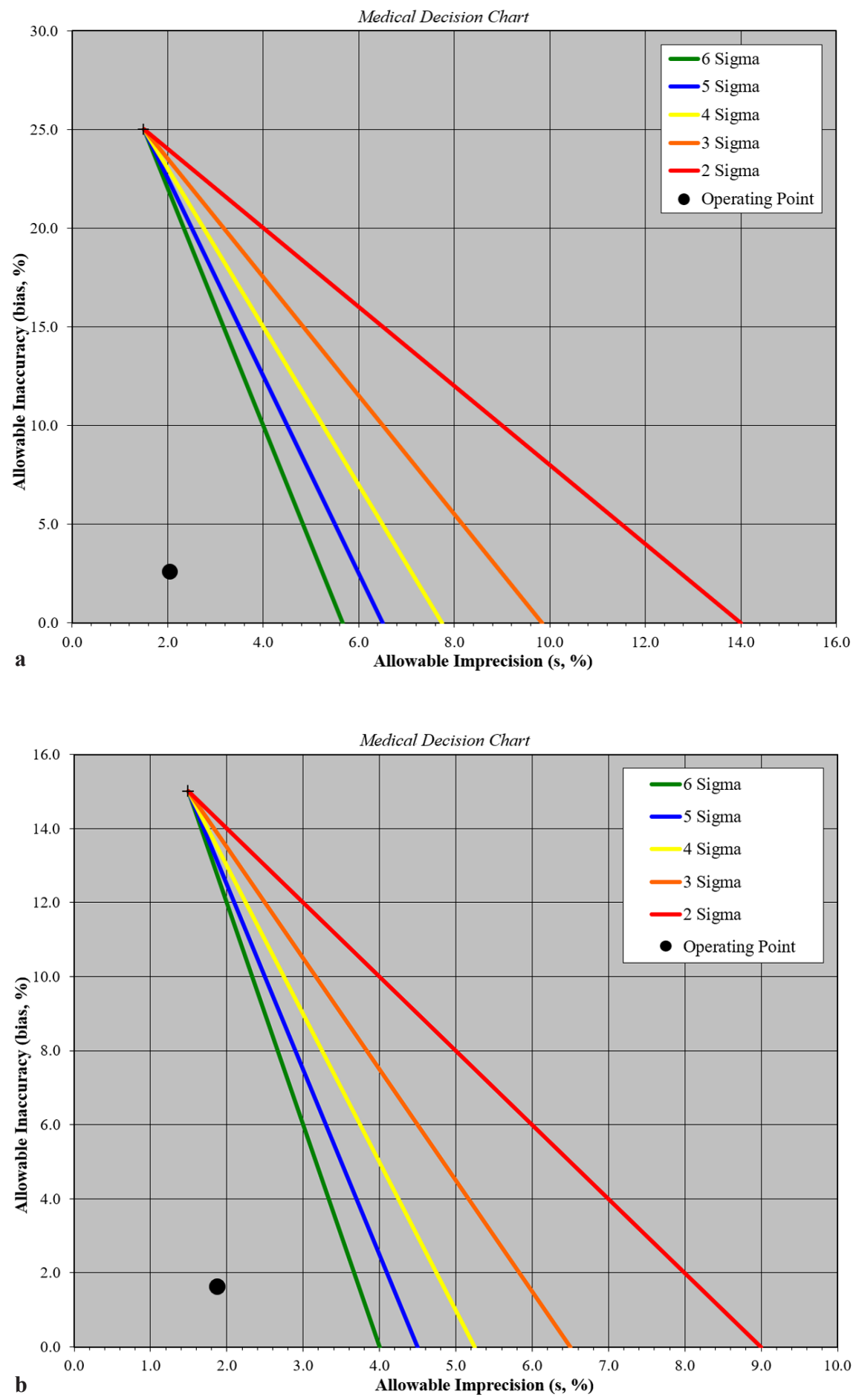

Figure 1. Sample figure for Sigma performance. Colored lines represent sigma scores and the black point represents our laboratory's performance. In figure 1a the best performer for Sysmex XT-1800i was PLT on Normal Level. In figure 1b best performer for Cell-Dyne Ruby instrument was WBC for normal level. 
Table 1a. Sigma values, CV\%, Bias\%, TE\% for Sysmex Xt-1800i instrument with both strategies of SQC for Normal Level ( 2 times a day every 12 hrs each level and 3 times a day every 8 hrs each level) and CV\% Desirable value and $\mathrm{TEa} \%$ requirements.

\begin{tabular}{|c|c|c|c|c|c|c|c|c|c|}
\hline \multirow[b]{2}{*}{ Analyte } & \multicolumn{2}{|c|}{ Sigma Value } & \multicolumn{2}{|c|}{$\mathrm{CV} \%$} & \multirow{2}{*}{$\begin{array}{c}\text { Bias\% } \\
\text { Both } \\
\text { Models }\end{array}$} & \multicolumn{2}{|c|}{ TE \% } & \multirow[b]{2}{*}{$\begin{array}{c}\text { CV\% } \\
\text { Desirabile }\end{array}$} & \multirow[b]{2}{*}{ ТЕа\% } \\
\hline & $\begin{array}{c}2 \times 3 \\
\text { Model }\end{array}$ & $\begin{array}{c}3 \times 3 \\
\text { Model } \\
\end{array}$ & $\begin{array}{c}2 \times 3 \\
\text { Model }\end{array}$ & $\begin{array}{c}3 \times 3 \\
\text { Model } \\
\end{array}$ & & $\begin{array}{c}2 \times 3 \\
\text { Model }\end{array}$ & $\begin{array}{c}\mathbf{3 x 3} \\
\text { Model } \\
\end{array}$ & & \\
\hline WBC & 6.97 & 5.23 & 1.45 & 1.93 & 4.9 & 7.29 & 8.08 & 5.5 & 15 \\
\hline HCT & 6.74 & 5.13 & 0.99 & 1.3 & 3.33 & 4.96 & 5.47 & 2.8 & 10 \\
\hline HGB & 10.13 & 5.21 & 0.55 & 1.07 & 1.43 & 2.33 & 3.19 & 2.8 & 7 \\
\hline PLT & 5.03 & 5.31 & 4.72 & 4.47 & 1.25 & 9.03 & 8.62 & 9.1 & 25 \\
\hline RBC & 9.19 & 8.08 & 0.58 & 0.66 & 0.67 & 1.62 & 1.75 & 1.6 & 6 \\
\hline
\end{tabular}

Table 1b. Sigma values, CV\%, Bias\%, TE\% for Sysmex Xt-1800i instrument with both strategies of SQC for High Level ( 2 times a day every $12 \mathrm{hrs}$ each level and 3 times a day every $8 \mathrm{hrs}$ each level) and CV\% Desirable value and $\mathrm{TEa} \%$ requirements.

\begin{tabular}{|c|c|c|c|c|c|c|c|c|c|}
\hline \multirow[b]{2}{*}{ Analyte } & \multicolumn{2}{|c|}{ Sigma Value } & \multicolumn{2}{|c|}{ CV\% } & \multirow{2}{*}{$\begin{array}{c}\text { Bias\% } \\
\text { Both } \\
\text { Models } \\
\end{array}$} & \multicolumn{2}{|c|}{ TE \% } & \multirow[b]{2}{*}{$\begin{array}{c}\text { CV\% } \\
\text { Desirabile }\end{array}$} & \multirow[b]{2}{*}{ ТЕa\% $\%$} \\
\hline & $\begin{array}{c}2 \times 3 \\
\text { Model }\end{array}$ & $\begin{array}{c}3 \times 3 \\
\text { Model }\end{array}$ & $\begin{array}{c}2 \times 3 \\
\text { Model }\end{array}$ & $\begin{array}{c}3 \times 3 \\
\text { Model }\end{array}$ & & $\begin{array}{c}2 \times 3 \\
\text { Model }\end{array}$ & $\begin{array}{c}3 \times 3 \\
\text { Model } \\
\end{array}$ & & \\
\hline WBC & 3.81 & 3.33 & 1.19 & 1.36 & 10.47 & 12.43 & 12.71 & 5.5 & 15 \\
\hline HCT & 4.88 & 4.94 & 0.81 & 0.8 & 6.05 & 7.38 & 7.37 & 2.8 & 10 \\
\hline HGB & 3.16 & 3.54 & 0.83 & 0.74 & 4.38 & 5.74 & 5.60 & 2.8 & 7 \\
\hline PLT & 9.8 & 10.89 & 2.29 & 2.06 & 2.56 & 6.33 & 5.95 & 9.1 & 25 \\
\hline $\mathrm{RBC}$ & 6.27 & 7.73 & 0.74 & 0.6 & 1.36 & 2.58 & 2.35 & 1.6 & 6 \\
\hline
\end{tabular}

Table 1c. Sigma values, CV\%, Bias\%, TE\% for Sysmex Xt-1800i instrument with both strategies of SQC for Low Level ( 2 times a day every 12 hrs each level and 3 times a day every 8 hrs each level) and CV\% Desirable value and $\mathrm{TEa} \%$ requirements.

\begin{tabular}{lccccccccc}
\hline \multirow{2}{*}{ Analyte } & \multicolumn{2}{c}{ Sigma Value } & \multicolumn{2}{c}{ CV\% } & Bias\% & \multicolumn{2}{c}{ TE \% } & \multirow{2}{*}{ CV\% } & \multirow{2}{*}{ TEa\% } \\
\cline { 2 - 8 } & $\begin{array}{c}\mathbf{2} \mathbf{3} 3 \\
\text { Model }\end{array}$ & $\begin{array}{c}\mathbf{3 x 3} \\
\text { Model }\end{array}$ & $\begin{array}{c}\mathbf{2 x 3} \\
\text { Model }\end{array}$ & $\begin{array}{c}\mathbf{3 x 3} \\
\text { Model }\end{array}$ & $\begin{array}{c}\text { Both } \\
\text { Models }\end{array}$ & $\begin{array}{c}\mathbf{2 x 3} \\
\text { Model }\end{array}$ & $\begin{array}{c}\mathbf{3 x 3} \\
\text { Model }\end{array}$ & Desirabile & \\
\hline WBC & 5.63 & 4.38 & 2.25 & 2.89 & 2.34 & 6.05 & 7.1 & 5.5 & 15 \\
\hline HCT & 7.51 & 10.43 & 1.25 & 0.9 & 0.61 & 2.67 & 2.09 & 2.8 & 10 \\
\hline HGB & 2.96 & 2.51 & 1.35 & 1.59 & 3.01 & 5.23 & 5.63 & 2.8 & 7 \\
\hline PLT & 2.31 & 2.22 & 10.19 & 10.59 & 1.51 & 18.32 & 18.98 & 9.1 & 25 \\
\hline RBC & 5.63 & 4.38 & 0.96 & 0.9 & 0.41 & 1.99 & 1.89 & 1.6 & 6 \\
\hline
\end{tabular}

levels of Sigma scores obtained for PLT on Sysmex XT-1800i and RBC on Cell-Dyne Ruby for Low-level QC are probably a consequence of the continuous degradation of the control material used from the first opening of the vials.

The Error detections estimated for these instruments were 0.84 for Sysmex XT-1800i and 0.53 for Cell-Dyne Ruby. In other words, each of them would detect the error in maximum two runs with three control points, but this detection rate would increase as the number of controls increases. Considering the Six Sigma values obtained in our laboratory, the "worst performer" for each instrument and the recommendations for the multirules that should be applied depending on Sigma value, a combination of the following rules is advisable: $13 \mathrm{~s} / 2$ of $32 \mathrm{~s} / \mathrm{R} 4 \mathrm{~s} / 31 \mathrm{~s} / 6 \mathrm{x}$ with $\mathrm{N}=6$. Since our laboratory reports patient results in continu- 
Table 2a. Sigma values, CV\%, Bias\% and TEa\% Values for Cell-Dyne Ruby Instrument with both startegies of SQC for Normal Level ( 2 times a day every 12 hrs each level and 3 times a day every 8 hrs each level).

\begin{tabular}{|c|c|c|c|c|c|c|c|c|c|}
\hline \multirow[b]{2}{*}{ Analyte } & \multicolumn{2}{|c|}{ Sigma Value } & \multicolumn{2}{|c|}{ CV\% } & \multirow{2}{*}{$\begin{array}{c}\text { Bias\% } \\
\text { Both } \\
\text { Models } \\
\end{array}$} & \multicolumn{2}{|c|}{ TE \% } & \multirow{2}{*}{$\begin{array}{c}\text { CV\% } \\
\text { Desirabile }\end{array}$} & \multirow[b]{2}{*}{ ТЕа\% } \\
\hline & $\begin{array}{c}2 \times 3 \\
\text { Model } \\
\end{array}$ & $\begin{array}{c}3 \times 3 \\
\text { Model } \\
\end{array}$ & $\begin{array}{c}2 \times 3 \\
\text { Model }\end{array}$ & $\begin{array}{c}3 \times 3 \\
\text { Model }\end{array}$ & & $\begin{array}{c}2 \times 3 \\
\text { Model }\end{array}$ & $\begin{array}{c}\mathbf{3 x 3} \\
\text { Model }\end{array}$ & & \\
\hline WBC & 6.32 & 7.12 & 2.12 & 1.88 & 1.61 & 5.11 & 4.71 & 5.5 & 15 \\
\hline HGB & 4.79 & 6.17 & 1.34 & 1.04 & 0.58 & 2.79 & 2.29 & 2.8 & 7 \\
\hline PLT & 4.03 & 5.18 & 5.37 & 4.18 & 3.34 & 12.2 & 10.23 & 9.1 & 25 \\
\hline $\mathrm{RBC}$ & 3.06 & 4.23 & 1.96 & 1.42 & 0.0 & 3.23 & 2.34 & 1.6 & 6 \\
\hline
\end{tabular}

Table 2b. Sigma values, CV\%, Bias\% and TEa\% Values for Cell-Dyne Ruby Instrument with both startegies of SQC for High Level (2 times a day every 12 hrs each level and 3 times a day every 8 hrs each level).

\begin{tabular}{|c|c|c|c|c|c|c|c|c|c|}
\hline \multirow[b]{2}{*}{ Analyte } & \multicolumn{2}{|c|}{ Sigma Value } & \multicolumn{2}{|c|}{$\mathrm{CV} \%$} & \multirow{2}{*}{$\begin{array}{c}\text { Bias\% } \\
\text { Both } \\
\text { Models }\end{array}$} & \multicolumn{2}{|c|}{ TE \% } & \multirow[b]{2}{*}{$\begin{array}{c}\text { CV\% } \\
\text { Desirabile }\end{array}$} & \multirow[b]{2}{*}{ ТЕa\% } \\
\hline & $\begin{array}{c}2 \times 3 \\
\text { Model }\end{array}$ & $\begin{array}{c}\mathbf{3 x 3} \\
\text { Model }\end{array}$ & $\begin{array}{c}2 \times 3 \\
\text { Model }\end{array}$ & $\begin{array}{c}\mathbf{3 x 3} \\
\text { Model }\end{array}$ & & $\begin{array}{c}2 \times 3 \\
\text { Model }\end{array}$ & $\begin{array}{c}\mathbf{3 x 3} \\
\text { Model }\end{array}$ & & \\
\hline WBC & 7.46 & 4.93 & 1.82 & 2.75 & 1.43 & 4.43 & 5.96 & 5.5 & 15 \\
\hline HGB & 5.63 & 6.9 & 1.14 & 0.93 & 0.58 & 2.46 & 2.11 & 2.8 & 7 \\
\hline PLT & 5.67 & 4.7 & 3.82 & 4.61 & 3.34 & 9.64 & 10.94 & 9.1 & 25 \\
\hline $\mathrm{RBC}$ & 3.37 & 3.77 & 1.78 & 1.59 & 0.0 & 2.94 & 2.62 & 1.6 & 6 \\
\hline
\end{tabular}

Table 2c. Sigma values, CV\%, Bias\% and TE\% Values for Cell-Dyne Ruby Instrument with both startegies of SQC for Low Level (2 times a day every 12 hrs each level and 3 times a day every 8 hrs each level).

\begin{tabular}{|c|c|c|c|c|c|c|c|c|c|}
\hline \multirow[b]{2}{*}{ Analyte } & \multicolumn{2}{|c|}{ Sigma Value } & \multicolumn{2}{|c|}{ CV\% } & \multirow{2}{*}{$\begin{array}{l}\text { Bias\% } \\
\text { Both } \\
\text { Models }\end{array}$} & \multicolumn{2}{|c|}{ TE \% } & \multirow{2}{*}{$\begin{array}{c}\text { CV\% } \\
\text { Desirabile }\end{array}$} & \multirow[b]{2}{*}{ ТЕа\% } \\
\hline & $\begin{array}{c}2 \times 3 \\
\text { Model }\end{array}$ & $\begin{array}{c}3 \times 3 \\
\text { Model }\end{array}$ & $\begin{array}{c}2 \times 3 \\
\text { Model }\end{array}$ & $\begin{array}{c}\mathbf{3 x 3} \\
\text { Model }\end{array}$ & & $\begin{array}{c}2 \times 3 \\
\text { Model }\end{array}$ & $\begin{array}{c}3 \times 3 \\
\text { Model }\end{array}$ & & \\
\hline WBC & 7.46 & 4.93 & 2.35 & 2.62 & 0.62 & 4.5 & 4.94 & 5.5 & 15 \\
\hline HGB & 5.63 & 6.9 & 1.53 & 1.06 & 0.53 & 3.05 & 2.27 & 2.8 & 7 \\
\hline PLT & 5.67 & 4.7 & 6.35 & 6.39 & 0.88 & 11.36 & 11.42 & 9.1 & 25 \\
\hline $\mathrm{RBC}$ & 3.37 & 3.77 & 1.98 & 1.81 & 0.0 & 3.27 & 2.98 & 1.6 & 6 \\
\hline
\end{tabular}

ous run mode, these controls should be bracketed every 50 patients $(\mathrm{N}=1)$ in order to maintain a probability of false rejection (PFR) of 0.001 (8). In an evaluation of Bull's algorithm, Cembrowski and Westgard (9) showed that for some hematology parameters (hemoglobin, MCV), usage of the algorithm is less sensitive than using commercial blood in detecting shifts of 2DS thus, for these parameters, 9 batches are necessary to detect this shift; however, for 3DS shifts in RBC, the error detection is $100 \%$. Since the commercial control is an unstable material, using aver- age of normals may increase error detection, at least for some parameters.

Since Cell-Dyne Ruby instrument has this function implemented by the manufacturer and it is recommended that no more than $10 \%$ of the population/ each batch to come from neonates, oncology, and haematology wards $(9,10)$, patients from the above-mentioned wards were run with the Sysmex XT-1800i instrument thus avoiding the influence on Average of Normal (AON) and MoA calculation. For Cell-Dyne Ruby instrument, commercial control and moving average were used together to assure an increased error 
detection. Moving average is a useful tool as a QC instrument, both in method stability evaluation and in assuring a continuous internal quality control, especially when the QC material has limited stability (11).

Much of the efforts made by laboratories are directed towards SQC or different QC strategies, but an important part of the total testing process remains out of the control of the laboratory (12) (13). It has been stated that around $70 \%$ of all errors occur during the preanalytical phase of the process, of which $73 \%$ of errors were classified as avoidable (14). The quality of the samples influences the results; while some of the defects are easy to identify (correct proportion between blood and anticoagulants, right type of tube, or the presence of large clots), other defects are not so easy to detect. The presence of micro-clots in samples is often detected only after a flag message is displayed by the instrument. Of 10,000 samples evaluated, in 168 (1.68\%) cases a "sampling error" flagging message appeared, meaning that not all samples with defects were correctly identified, this percentage corresponding to a Sigma score of 3.4. Sigma scores for defects that are correctly identified and for those defects that remain undetected by laboratory staff were similar.

In a study concerning hemolysis evaluation with a visual scale and an automated hemolysis test, visual inspection overestimated the hemolysis rate in coagulation sample tubes (15). Both clinicians and laboratory staff should ensure the right samples are used for the correct patient investigation.

Another aspect of the total testing process concerns the post-analytical phase. Although results are released in LIS in 13 minutes on average, and in emergency cases sometimes in 2 minutes from the moment the samples are received in the laboratory, some samples take a much longer time to arrive in the laboratory. In some cases even if results are released in LIS, clinicians are not aware of the results immediately, this delay in reviewing the results will falsely increase the TAT (16).

\section{Conclusions}

Given the performances of the total testing process implemented for CBC in our laboratory, performing one level of internal control after every 50 samples/batch seems to fulfill both the HMO $1301 / 2007$, regarding the frequency, and of the 1SO 15189:2013 recommendation concerning the number of control levels analyzed during 24 hrs. In this way, in 24 hrs all three levels of control would be assayed at least once. Each laboratory should establish its own internal quality control scheme, following its performance and operating mode.

Considering that all phases of the total testing process are subject to errors, clinicians and laboratories should be more diligent in detecting these errors. Although most of laboratory errors occur during the preanalytical phase, laboratories must be aware that the preanalytical aspects fall under their responsibility. In this regard, laboratory staff must continue, and make even greater efforts to achieve a higher quality in the preanalytical phase. In some cases, laboratories must use multiple instruments of quality assessment, solely one being insufficient for achieving the desired goal. All quality instruments must work together to assure better patient results and each laboratory should design its own control plan that is appropriate for better quality achievement.
Abbreviations
SQC - statistical quality control
QC - quality control
CBC - complete blood count
CLIA - Clinical Laboraory Improvement Amen- demnets
HMO - Health Ministry Order
$\mathrm{N}$ - number of control points 
TE - Total Error of the method

$\mathrm{CV} \%$ - coefficient of variation

PT - Proficiency Testing

SD - Standard Deviation

TEa - Total Allowable Error

MoA - Moving Average

EDTA - ethylene-diamine -tetraacetic acid

LIS - Laboratory Informational System

DPMO - defects per million of opportunities

TAT - turnaround time

PLT - platelets

RBC - red blood cells

WBC - white blood cells

HGB - hemoglobin

HCT - hematocrit

\section{Acknowledgments}

This work was funded by the Romanian Association of Medical Laboratories (AMLR) 2018 research grant.

\section{Authors' contribution}

ORO: investigation, methodology, analysis, evaluation, writing the original draft;

$\mathrm{AH}$ : project administration, resources, supervision, writing-review \& editing;

OP: data editing, data analysis;

DK: data analysis;

MD: reviewing the manuscript.

\section{Conflict of interest}

Nothing to disclose.

\section{References}

1. www.ASRO.ro (Asociatia de Standardizare din Romania) Standard SR EN ISO 15189:2013. 2013:37.

2. Statland BE. Medical decision level. Westgard QC Inc. Basic QC Practices, 4th Edition. Madison WI. 2016:112-6.

3. Westgard JO. Complying with CLIA QC Regulations. Westgard QC Inc. Basic QC Practices, 4th Edition,
Madison WI. 2016:171-88.

4. Westgard JO. Analytical Performance Capability. Westgard QC Inc. Six Sigma Quality Design and Control, 2nd Editiond, Madison WI. 2016:45.

5. CLSI. User Verification of Precision and estimation of Bias; Approved Guideline-Third Edition CLSI document EP15-A3.Wayne, PA:Clinical and laboraty Standards Institute;2014.

6. Bull BS, Elashoff RM, Heilbron DC, Couperus J,A. Study of various estimators for the derivation of quality control procedures from patient erytrocite indices. Am J Clin Pathol. 1974;61(4):473-81. DOI: 10.1093/ ajcp/61.4.473

7. Parvin CA. Assesing the impact of the frequency of quality control testing on the quality of reported patient results. Clin Chem. 2008;4(12):2049-54. DOI: 10.1373/clinchem.2008.113639

8. Yago M. Selecting Statistical Quality Control Procedures for Limiting the Impact of Increases in Analytical Random Error on Patient Safety. Clin Chem. 2017;63(5):1022-30. DOI: 10.1373/ clinchem.2016.267682

9. Cembrowski GS, Westgard JO. Quality control of multichannel hematology analyzers: evaluation of Bull's algorithm. Am J Clin Pathol. 1985;83(3):337-45. DOI: 10.1093/ajcp/83.3.337

10. Westgard JO, Smith FA, Mountain PJ, Boss S. Design and assessment of average of normals patient data algorithms to maximize run lenghts for automatic process control. Clin Chem 1996;42(10):1683-88. DOI: 10.1093/clinchem/42.10.1683

11. van Rossum H. Moving average quality control: principles, practical application and future perspectives. Clin Chem Lab Med. 2019;57(6):773-82. DOI: 10.1515/ cclm-2018-0795

12. Vis JY, Huisman A. Verification and quality control of routine hematology analyzers. Int $\mathrm{J}$ Lab Hematol. 2016;38 (suppl 1):100-9. DOI: 10.1111/ijlh.12503

13. Lewis SM. Quality control: good laboratory practice - an overview of quality assurance. WHO/LAB/98.4 Available at https://apps.who.int/iris/handle/ (last access 09/04/2019)

14. Carraro P, Plebani M. Errors in a stat laboratory:types and frequencies 10 years later. Clin Chem. 2017; 53(7):1338-42. DOI: 10.1373/clinchem.2007.088344

15. Binzari E, Zaharia M, Barbu S, Oprea OR, Dobreanu $\mathrm{M}$ Hemolysis has no influence on routine coagulation tests without anticoagulant therapy-a referral Romanian emergency hospital laboratory experience. Rev Romana Med Lab. 2019; 27 (4):375-82. DOI: 10.2478/ rrlm-2019-0034

16. Hawkins RC. Laboratory Turnaround Time. Clin Biochem Rev. 2007;28(4):179-94. 